\title{
Possible Magnetic Activity in the Low Mass X-ray Binary EXO 0748-676
}

\author{
Michael T. Wolff ${ }^{1}$, Kent S. Wood ${ }^{2}$ \\ and \\ Paul S. Ray ${ }^{3}$
}

\begin{abstract}
We report evidence of magnetic activity associated with the secondary star in the EXO 0748-676 low mass X-ray binary system. An analysis of a sequence of five consecutive X-ray eclipses observed during December 2003 with the RXTE satellite brings out a feature occurring during ingress we interpret as the X-ray photoelectric absorption shadow, as seen by an observer at Earth, of a plasma structure suspended above the surface of the secondary star. The light curve feature consists of an initial drop in count rate to near zero (the absorption shadow) with a very short rebound to a significant fraction of the pre-ingress count rate and then a final plunge to totality over a total time scale of $\sim 25 \mathrm{~s}$. The ingress feature persists for at least 5 consecutive orbital periods (a total of $\sim 19$ hr), and possibly up to 5 days in our data. Our data also show significant postegress dipping during this eclipse sequence, unusual for this source, indicating possible secondary star mass ejection during this episode.
\end{abstract}

Subject headings: X-rays: binaries, binaries: eclipsing, stars: individual (EXO 0748-676), stars: magnetic fields

\section{Introduction}

EXO 0748-676 is the only persistently active low mass X-ray binary (LMXB) that displays full X-ray eclipses. In order to observe orbital period variations in a LMXB we have

\footnotetext{
${ }^{1}$ Space Science Division, Naval Research Laboratory, Washington, DC 20375; Michael.Wolff@nrl.navy.mil

${ }^{2}$ Space Science Division, Naval Research Laboratory, Washington, DC 20375; Kent.Wood@nrl.navy.mil

${ }^{3}$ Space Science Division, Naval Research Laboratory, Washington, DC 20375; Paul.Ray@nrl.navy.mil
} 
conducted a long-term observational program (see Wolff et al. 2002, and references therein) of monitoring the X-ray eclipses of EXO0748-676 ( $P_{\text {orb }}=3.82 \mathrm{hr}$ ) with the Rossi X-Ray Timing Explorer (RXTE) satellite. By monitoring the mid-eclipse times we can investigate changes in the orbital period and constrain the magnitudes of the physical processes that cause the observed changes, allowing estimation of their long-term evolutionary effects.

In this letter we report on a chance observational circumstance that has allowed us to identify a possible X-ray signature of magnetic activity in the secondary star in EXO 0748-676. Magnetic activity has been observed for some time in both the white dwarfs and secondary stars of cataclysmic variable systems (e.g., Warner 1995; Washuettl \& Strassmeier 2001). To our knowledge, however, magnetic activity associated with the secondary stars of neutron star or black hole candidate LMXB systems has yet to be reported. Indeed, the secondary star in the EXO 0748-676 system has never been reliably observed because of the dominance of the optical emission by the accretion disk (Schmidtke \& Cowley 1987; Pearson et al. 2006). Magnetic fields associated with the companion star may lead to modulation of rates of angular momentum exchange and mass transfer between the binary components and changes in the structure of the secondary star (Tauris \& van den Heuvel 2006). Estimates of the magnitude of magnetic effects on LMXB evolution have been hampered by the lack of observations of magnetic activity in real systems that can be tied directly to the observable properties of the secondary star. This is because there are few systems where distinct evolutionary processes can be directly observed (e.g., observing orbital evolution by timing eclipses) and even in systems where such observations are possible the nature of these processes is controversial (e.g., see discussion of several eclipsing LMXBs in Wolff et al. 2002). Finally, in analogy with the 11-year solar cycle, magnetic fields associated with the secondary star in a LMXB could show activity cycles (Lanza et al. 1998), and may also cause observable transient phenomena.

\section{RXTE Observations}

The observations we report here were all made with the Proportional Counter Array (PCA) on the RXTE. The PCA is an array of five large-area X-ray proportional counters (Proportional Counter Units or PCUs) with microsecond timing accuracy (Jahoda et al. 2006). From the beginning of the RXTE mission (1996) we have monitored EXO 0748-676 to time X-ray eclipses with sub-second accuracy. This monitoring is organized into "campaigns" consisting of 4-6 separate observations of EXO 0748-676 over roughly one day with one or two month spacing between campaigns. Our timing accuracy for each eclipse is limited by counting statistics in the X-ray flux and any intrinsic variability in the X-ray emission. 
Eclipse cycle $(N)$ is determined by the numbering system of Parmar et al. (1991) with the updated ephemeris: $T_{0}(\mathrm{TDB} ; \mathrm{MJD})=46111.0751910$ and $P_{\text {orb }}=0.15933778478$ days. We record full photon event data using GoodXenon mode. For each eclipse light curve we select only layer 1 photon events from those PCUs that are on during the entire observation. This allows us to minimize the noise in background-subtracted light curves at very low count rates (e.g., at eclipse totality where the source count rate is near zero). However, below we will have occasion to refer to the hardness of the source spectrum and in those situations we select events from all layers allowing us to bring out more clearly the spectral character at energies above $7 \mathrm{keV}$ of the ingress feature we study. Because of improvements in the PCA background model we extract events in the energy range $2-20 \mathrm{keV}$, rather than $2-12$ $\mathrm{keV}$ as done in Wolff et al. (2002) We utilize the combined background model released on August 6, 2006 available at the RXTE web sit 1 . Each $R X T E$ observed eclipse is processed using the FTOOLS data analysis package. See Wolff et al. (2002) for further details of our analysis procedure.

\section{Observed Eclipse Profiles}

Of primary interest to us here are five sequential eclipses, $N=43095$ through $N=$ 43099, observed by RXTE on December 4-5, 2003. These are listed in Table 1 where we also include for comparison eclipses occurring both before and after this sequence. The ingress profiles for these five eclipses are shown in Figure 1 along with, for comparison, the ingress of an eclipse observed roughly 93 days later $(N=43684)$. All five eclipse ingresses $(N=43095-43099)$ have an unusual structure, namely, as X-ray eclipse first contact is approached, the count rate first goes down, then rebounds to a significant fraction of the pre-ingress level (we refer to this as the ingress "spike"), then drops to a count rate of essentially zero as real totality begins. Contrast this behavior with the eclipse shown in the bottom panel of Figure $1(N=43684)$. In that case the ingress is sharp, lasting only about $2-3$ seconds whereas in each of the $N=43095-43099$ eclipses the complete ingress structure lasts at least $25 \mathrm{~s}$. This spike feature lasts for at least five orbits ( 19 hr) as shown in Figure 1. Also, the ingress spikes occur at the same phase of the orbit relative to the occulting edge of the secondary for each $N=43095-43099$ eclipse. The physical cause of the light curve spike occurs on the same side of the secondary for each of the five orbits. Furthermore, examining eclipse $N=43065$ we see the ingress comes $\sim 10$ s early compared to a local constant period ephemeris, a possible sign of the emergence of the loop from the

${ }^{1} \mathrm{PCA}$ X-ray background model information is found at the web site
http://rxte.gsfc.nasa.gov/docs/xte/pca_news.html.


stellar interior before its geometrical structure allows X-rays to partially transit through it on their way to the Earth.

The energy distribution of the photons in the ingress spike is "hard" in that the ratio of hard $(E>7 \mathrm{keV})$ to soft $(E<7 \mathrm{keV})$ count rate goes up in the spike feature and the extended ingress, as can again be seen in Figure 1. Indeed, above $7 \mathrm{keV}$ the eclipse ingress, while somewhat disorganized, occurs on the totality-side of the spike. In other words, the initial count rate drop occurs because of soft photons being removed from the X-ray beam along our line of sight. This is a signature of photoelectric absorption by material moving ahead of the occulting edge of the secondary star.

Another feature of this set of eclipses is a persistent level of post-egress dipping in the PCA energy band shown in Figure 2. Based on our experience from the eclipse monitoring program, even though the PCA energy band is relatively hard, dipping before ingress is not unusual. In fact, dipping at all phases of the EXO 0748-676 orbit has been reported by several authors (e.g., Church et al. 1998; Sidoli et al. 2005) but dipping of this magnitude at the immediate post-egress orbit phase is relatively rare. Examining the spectral fits to the post-egress PCA data shows, assuming solar abundances, the inferred hydrogen column density is $n_{H} \sim 10^{23} \mathrm{~cm}^{-2}$ for these post-eclipse data. Thomas et al. (1997) found from $A S C A$ observations of EXO 0748-676 that $n_{H}$ varied from $4 \times 10^{21} \mathrm{~cm}^{-2}$ outside of any dipping to a high of $10^{23} \mathrm{~cm}^{-2}$ during deep dips. After the five eclipse sequence of interest the observed column density in our post-egress spectral fits goes down by more than an order of magnitude and the strong post-egress dipping ceases.

\section{Discussion}

The spiked ingress behavior described above can occur if, as the line of sight from the neutron star to the Earth comes close to the occulting edge of the secondary star, a highdensity plasma structure suspended above the surface of the secondary and in the plane of the line of sight, absorbs X-ray photons out of the beam. Then, a few seconds later the $\mathrm{X}$-ray line of sight passes through a lower density region between the suspended structure and the true occulting edge of the secondary star, creating the brief spike in the light curve. This eclipse geometry is schematically shown in Figure 3. Such a structure might be similar to a magnetic loop in the solar atmosphere that is anchored at two foot points of opposite magnetic polarity (e.g., Brković et al. 2002).

Structures of the sort we propose may last for several rotations of the secondary star because the secondary's magnetic field along with the relatively gentle gravitation potential 
gradient will maintain the mechanical rigidity of the structure. Utilizing Kepler's law and the EXO 0748-676 orbital period of $P_{\text {orb }}=3.82 \mathrm{hr}$, we obtain $A=1.4\left(\frac{M_{1}}{1.4 M_{\odot}}\right)(1+q)^{1 / 3} R_{\odot}$, for the binary separation, where $q=M_{2} / M_{1}$ is the binary mass ratio and $M_{1}$ is the compact object mass. We assume component (1) is a neutron star and set $M_{1}=1.4 M_{\odot}$. If $M_{2}=$ $0.4 M_{\odot}$ (Parmar et al. 1986) then $q=0.286$ and $A=1.5 R_{\odot}$. The radius of the secondary star, $R_{2}$, is the radius of the Roche lobe surrounding it (Eggleton 1983),

$$
R_{2}=\frac{0.49 q^{2 / 3}}{0.6 q^{2 / 3}+\ln \left(1+q^{1 / 3}\right)} A \sim 0.42 R_{\odot},
$$

for the above $A$ and $q$. An estimate of the width of the feature is $w \sim \frac{10 s}{\frac{1}{2} \Delta T_{e c}} \times R_{2} \sim$ $0.0084 R_{2} \sim 5.9 \times 10^{8} \mathrm{~cm}$ where $\Delta T_{e c}$ is the average duration of eclipse totality $(495 \mathrm{~s}), 10 \mathrm{~s}$ is the time scale of the drop before the spike feature, and where we have assumed that the system inclination is $i \geq 75^{\circ}$ (Parmar et al. 1986). From Figure 1 the count rate goes down $75 \%$ during the initial drop before the spike. Simulating the EXO 0748-676 pre-ingress spectrum to determine the necessary column density to make the $2-7 \mathrm{keV}$ flux drop by this amount yields a column density $N_{H} \sim 2.5 \times 10^{23} \mathrm{~cm}^{-2}$ implying a hydrogen density $n_{H} \sim$ $N_{H} / w \sim 4 \times 10^{14} \mathrm{~cm}^{-3}$ for the loop structure. This density is higher than the corresponding densities in solar magnetic loops $\left(n_{H} \sim 10^{9} \mathrm{~cm}^{-3}\right.$; Brković et al. 2002) and even higher than coronal densities observed in the G5III flare system Capella $\left(n_{H} \sim 4 \times 10^{11}-10^{13} \mathrm{~cm}^{-3}\right.$; Dupree et al. 1993). The secondary mass we use above $\left(M_{2}=0.4 M_{\odot}\right)$ corresponds to either a late K or early $\mathrm{M}$ type main sequence star. Such stars can have magnetic activity if they rotate and if they have significant convective envelopes (Hartmann \& Noyes 1987). The fact that EXO 0748-676 secondary rotates very fast (assuming it rotates synchronously) and that stars in this mass range are thought to have significant convective layers does support our magnetic loop hypothesis.

Figure 2 shows that post-egress dipping is prominent during the five eclipse sequence we are studying. This post-egress dipping could indicate significant mass ejection that moves away from the secondary accompanying the magnetic activity. Such mass ejections could be similar to coronal mass ejections that sometimes accompany solar magnetic activity. Because we observe only a total column density, however, we can not establish whether this material is associated with the accretion disk inside the neutron star Roche lobe, or it is outside the neutron star Roche lobe and moving away radially from the secondary.

In the simple model of Landini \& Landi (2002), in order for the structures to last in the solar corona they must be in hydrostatic equilibrium, internal velocities within the loop must be negligible, pressure gradients should be small, and energy dissipation processes within the loop must be uniform. Because the loop structure resides near the surface of the secondary star it will be near a saddle point in the Roche gravitational potential function. Thus, the 
effective force on a plasma structure will be small near the surface of the secondary. Second, if the forces on the loop are small then, unless there is significant non-uniformity in the energy dissipation or heating processes along the loop, velocities should be negligibly small and any pressure gradients should also be small. However, heating and cooling processes within the loop are difficult to characterize. The loop may have a hydrogen-poor abundance reflecting the abundance of the secondary star. Also, the loop will be bathed in substantial $\mathrm{X}$-rays from the accretion onto the neutron star, the radiation from an optically thin coronal plasma (if one exists), and from the radiation emerging from the stellar photosphere. At a density of $n_{H} \sim 4 \times 10^{14} \mathrm{~cm}^{-3}$ the loop may be optically thick, making its energy balance particularly difficult to determine.

The set of anomalous eclipses runs from cycle $N=43095$ to $N=43099$. Based on the $\mathrm{O}-\mathrm{C}$ residuals there is considerable intrinsic period jitter of the type studied in Hertz et al. (1997) and Wolff et al. (2002) prior to the spike eclipses. Other than this jitter, however, both before and after this set of observations the eclipses appear to be normal in that both the ingresses and egresses are well behaved although some eclipses have longer than average duration. However, the eclipses running from $N=43684$ to $N=44363$, in particular the set $N=43684-43687$ (Table 1), after the ingress feature disappears, are among the most stable eclipses we have observed from EXO 0748-676 with RXTE over our entire monitoring program. The emergence of the ingress magnetic loop and significant post-eclipse dipping may signify a dramatic transient event followed by quiescence in the system. The stable eclipses $N=43684-43687$ occur during the first of a series of luminosity excursions observed by the $R X T E$ All Sky Monitor that have continued on and off to the present day. During those excursions the EXO 0748-676 X-ray brightness can increase from its average of $\sim 8$ mcrab by factors of $2-5$ for weeks at a time. Magnetic activity in the secondary star may be modulating the mass accretion rate onto the neutron star and affecting the system X-ray luminosity.

\section{Conclusions}

We have reported on a sequence of five consecutive X-ray eclipses observed by $R X T E$ during December 2003 from the EXO 0748-676 LMXB system that show a repeating feature during ingress transitions. This feature is an anomalous X-ray peak during ingress we interpret as X-rays emitted from near the neutron star passing underneath a rigid plasma structure suspended above the surface of the secondary. This loop structure may be similar to magnetic loops suspended above the solar photosphere. This conclusion is based on the ingress feature lasting for at least five orbit periods and perhaps as long as five days, although 
this latter conclusion depends on the interpretation of one ingress profile. The occurrence of this ingress feature may be an indication of a transient event of significant magnitude in the secondary star since the $\mathrm{O}-\mathrm{C}$ residuals around the time of the event show the mid-eclipse timings wandering before the ingress feature appears but settling down after the ingress feature disappears. During this sequence of eclipses we also observed significant post-egress dipping which, as we noted, is unusual. This suggests that significant mass may have been liberated from the secondary star (similar to a coronal mass ejection) as part of this transient event. The occurrence of the X-ray spike on the limb of the secondary at the same orbit phase in sequential eclipses is consistent with the conventional picture of synchronous rotation as suggested by a number of investigations (e.g., Verbunt \& Phinney 1995). Magnetically maintained features playing a role in determining the eclipse occulting edge geometry could lead to significant departures from symmetry between ingress and egress. This may be the cause of the intrinsic jitter identified by Hertz et al. (1997) and Wolff et al. (2002). If true then the magnetic field of the secondary may perturb the structure of the secondary star, moderating both mass transfer and angular momentum exchange.

We thank Drs. Paul Hertz, Gerald Share, and J. Martin Laming for useful discussions and Evan Smith for help with RXTE scheduling. We thank an anonymous referee for critically reading the manuscript and many helpful suggestions. This work was supported by the NASA RXTE Guest Observer Program and the Office of Naval Research.

\section{REFERENCES}

Brković, A., Landi, E., Landini, M., Rüedi, I., \& Solanki, S. K. 2002, A\&A, 383, 661

Church, M. J., Balucinska-Church, M., Dotani, T., \& Asai, K. 1998, ApJ, 504, 516

Dupree, A. K., Brickhouse, N. S., Doschek, G. A., Green, J. C., \& Raymond, J. C. 1993, ApJ, 418, L41+

Eggleton, P. P. 1983, ApJ, 268, 368

Hartmann, L. W., \& Noyes, R. W. 1987, Ann. Rev. Astr. Astrphys., 25, 271

Hertz, P., Wood, K. S., \& Cominsky, L. R. 1997, ApJ, 486, 1000

Jahoda, K., Markwardt, C. B., Radeva, Y., Rots, A. H., Stark, M. J., Swank, J. H., Strohmayer, T. E., \& Zhang, W. 2006, ApJS, 163, 401

Landini, M., \& Landi, E. 2002, A\&A, 383, 653 
Lanza, A. F., Rodono, M., \& Rosner, R. 1998, MNRAS, 296, 893

Parmar, A. N., Smale, A. P., Verbunt, F., \& Corbet, R. H. D. 1991, ApJ, 366, 253

Parmar, A. N., White, N. E., Giommi, P., \& Gottwald, M. 1986, ApJ, 308, 199

Pearson et al. 2006, ApJ, 648, 1169

Schmidtke, P. C., \& Cowley, A. P. 1987, Astron. J., 93, 374

Sidoli, L., Parmar, A. N., \& Oosterbroek, T. 2005, A\&A, 429, 291

Tauris, T. M., \& van den Heuvel, E. P. J. 2006, in Compact Stellar X-ray Sources, ed. W. H. G. Lewin \& M. van der Klis (Cambridge University Press), 623

Thomas, B., Corbet, R., Smale, A. P., Asai, K., \& Dotani, T. 1997, ApJ, 480, L21

Verbunt, F., \& Phinney, E. S. 1995, A\&A, 296, 709

Warner, B. 1995, Cataclysmic variable stars (Cambridge Astrophysics Series, Cambridge, New York: Cambridge University Press)

Washuettl, A., \& Strassmeier, K. G. 2001, A\&A, 370, 218

Wolff, M. T., Hertz, P. L., Wood, K. S., Ray, P. S., \& Bandyopadhyay, R. M. 2002, ApJ, 575,384 


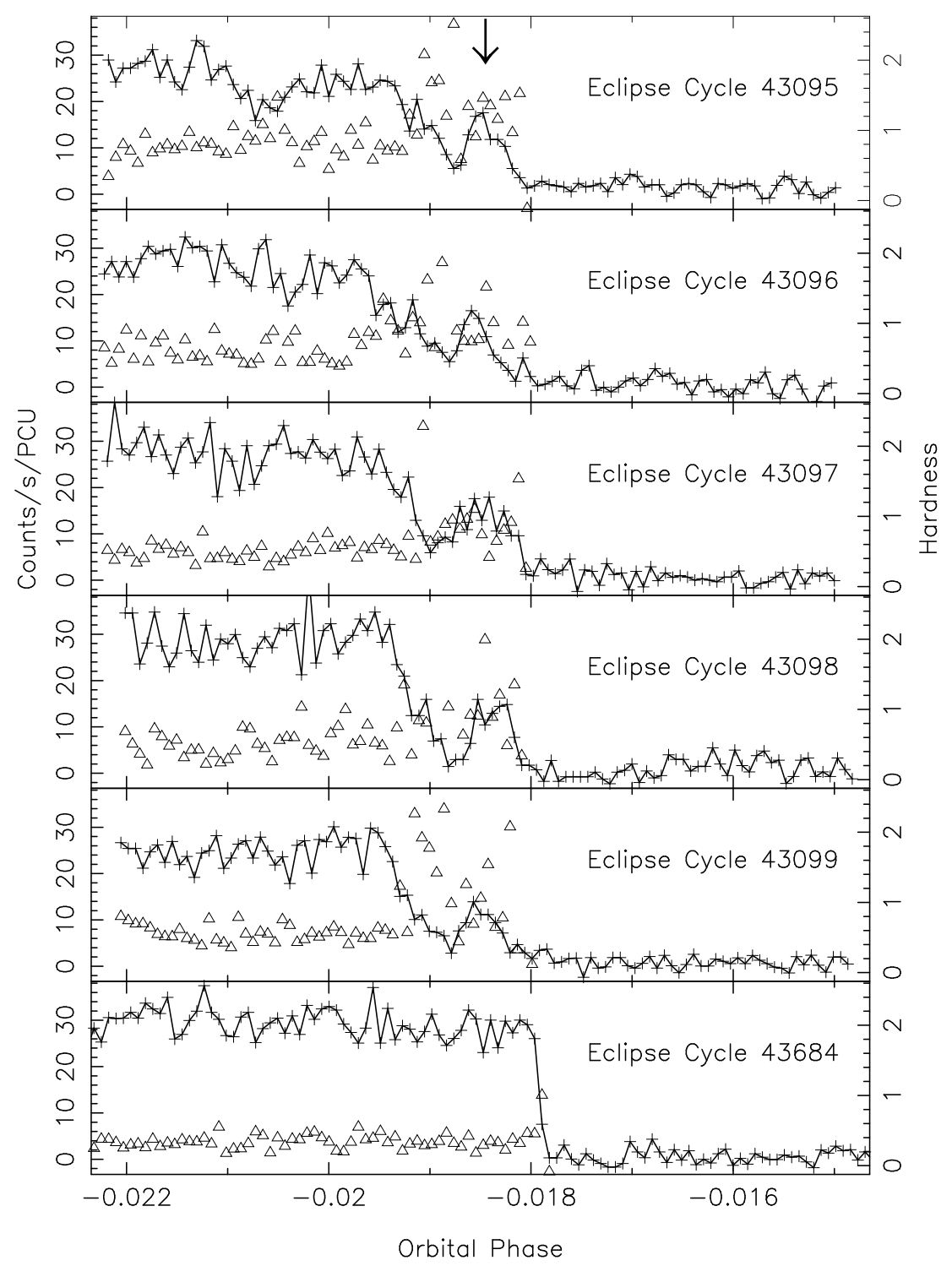

Fig. 1.- Six panels that show the light curves (crosses) and hardness ratios (triangles) of the five spiked eclipse ingresses (top five panels), and one particularly sharp eclipse $(N=43684)$ observed 93 days later. Phase zero is set by the mid-eclipse time for eclipse $N=43684$ and then applied to the other eclipses so that all ingress points can be seen phased to the same local ephemeris. The light curve points are the PCA count rate per PCU in $0.5 \mathrm{~s}$ time bins for the $2-20 \mathrm{keV}$ energy band and are connected with lines to allow them to be easily distinguished. The hardness is the ratio of the count rate in the $7-20 \mathrm{keV}$ energy band to that in the $2-7 \mathrm{keV}$ band. Inside eclipse totality hardness points are not plotted. The spike we discuss is centered at phase $\phi=-0.0185$ (downward arrow) while the real X-ray ingress appears to start $8 \mathrm{~s}$ later at phase $\phi=-0.0179$. 


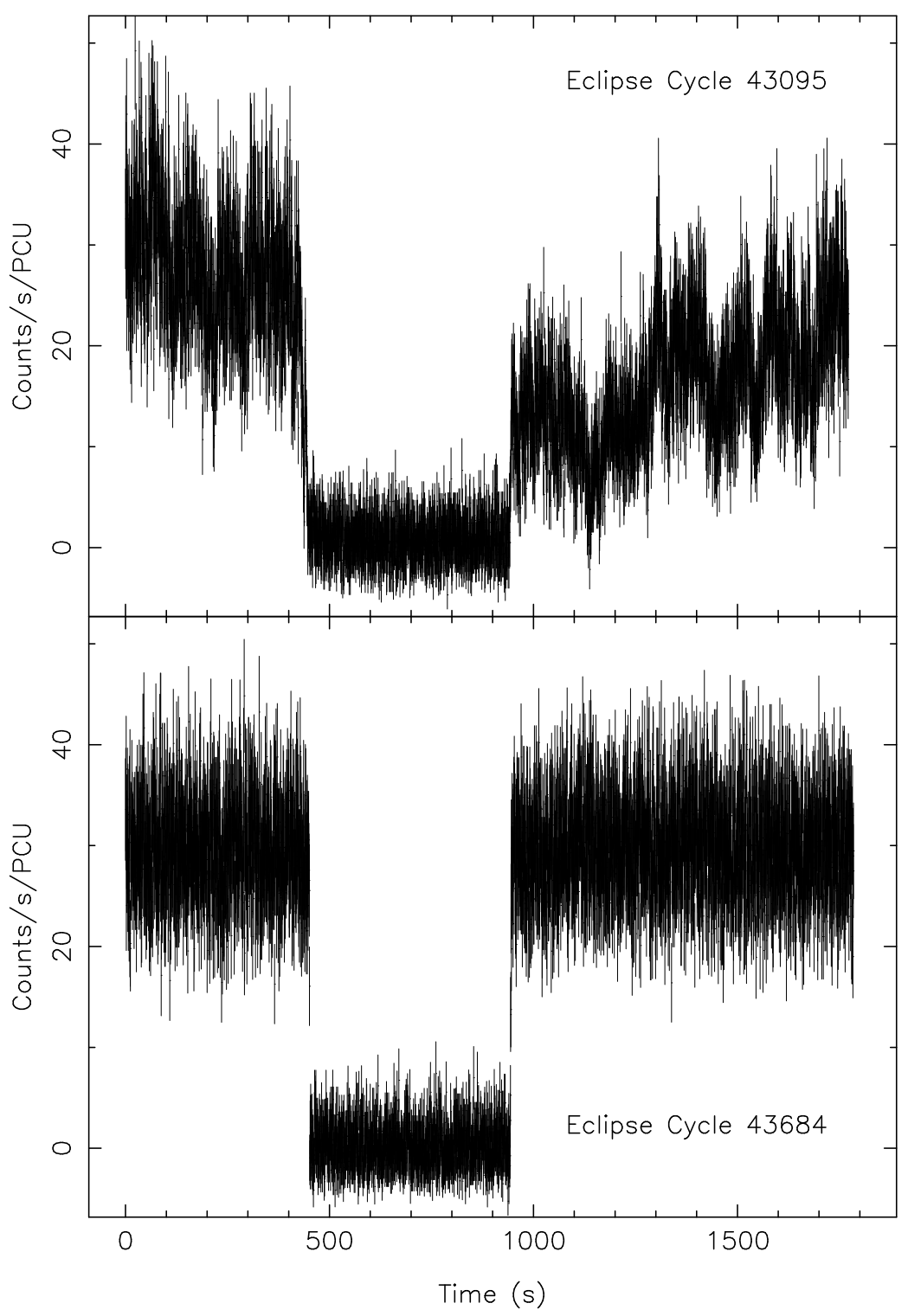

Fig. 2. - The light curves for the 2-20 keV energy band for the region around the eclipses $N=43095$ (top panel), and $N=43684$ (bottom panel). Eclipse $N=43095$ is the first of the eclipses with ingress spikes and eclipse $N=43684$ occurs three months later. The binary orbit phasing is arbitrary, only layer 1 events are included with time binning $0.5 \mathrm{~s}$. Note the strong dipping after $N=43095$ eclipse egress whereas the post-egress count rate is steady for the $N=43684$ eclipse. Our experience is that this level of post-egress dipping is rare during our monitoring observations. The count rate in each time bin is plotted with $\pm 1 \sigma$ error bars and the time scale is compressed relative to Figure 1 hence the ingress spike feature shown in Figure 1 does not stand out as strongly in the upper panel of this figure. 


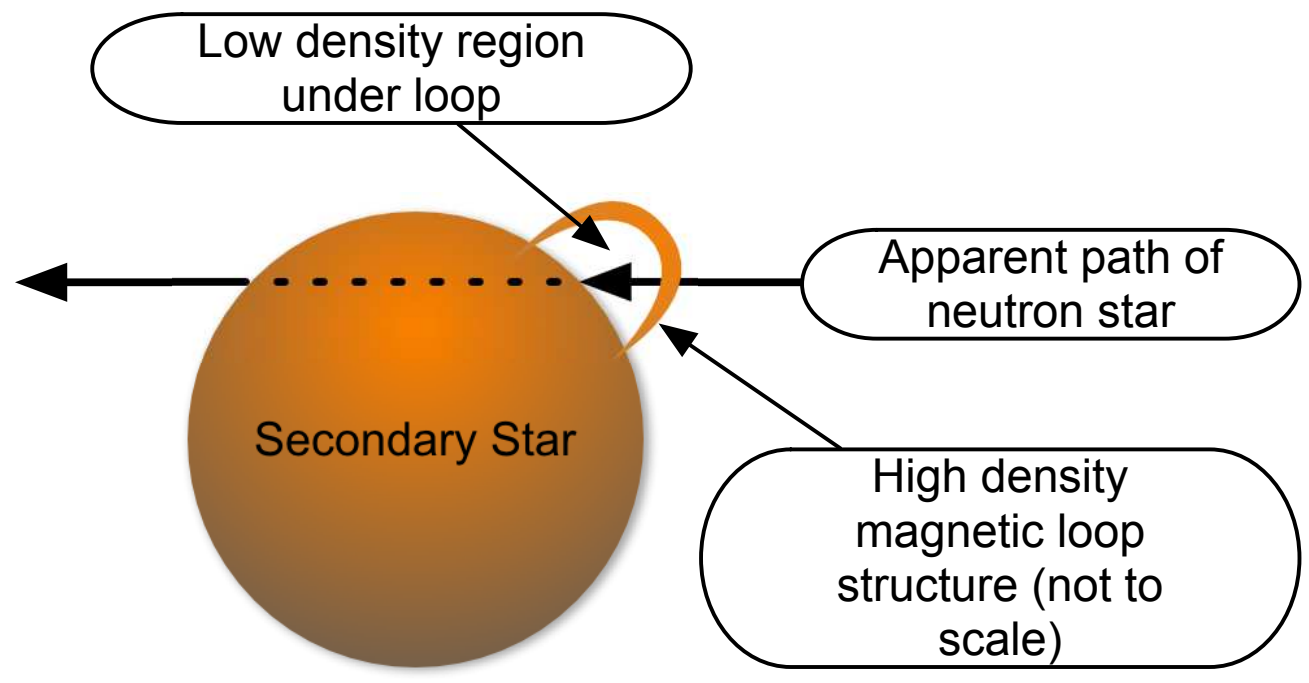

Fig. 3.- A schematic drawing of our model for the "spike" eclipses from the point of view of an observer at Earth. The spike in the ingress profile corresponds to when the apparent neutron star position is between the loop and the edge of the secondary star and shinning through the lower density material under the loop. True eclipse totality begins as the apparent position of the neutron star moves completely behind the secondary star and lasts until neutron star re-emergence.

\section{Table 1. RXTE Timing of Selected Full EXO 0748-676 X-ray Eclipses}

\begin{tabular}{cccc}
\hline \hline RXTE ObsID & Eclipse Cycle & $\begin{array}{c}\text { Fitted Mid-Eclipse Time } \\
\text { (MJD;TDB) }\end{array}$ & Comment \\
\hline $70048-13-12-00$ & 42716 & $52917.347911(3)$ & Normal eclipse. \\
$70048-13-13-00$ & 42717 & $52917.507251(5)$ & Burst partially obscures post-egress light curve. \\
$70048-13-14-00$ & 42718 & $52917.666590(6)$ & Normal eclipse. \\
$70048-13-15-00$ & 42719 & $52917.825925(5)$ & Normal eclipse; Some post-egress dipping. \\
$80040-01-07-00$ & 43065 & $52972.956764(5)$ & Apparent eclipse duration 505 s. \\
$70048-13-16-00$ & 43095 & $52977.736972(7)^{2}$ & Double-peaked ingress; Post-egress dipping. \\
$70048-13-17-00$ & 43096 & $52977.89628(1)^{2}$ & Double-peaked ingress; Post-egress dipping. \\
$70048-13-18-00$ & 43097 & $52978.055689(4)^{2}$ & Double-peaked ingress; Post-egress dipping. \\
$70048-13-19-00 G$ & 43098 & $52978.214950(7)^{2}$ & Double-peaked ingress; Post-egress dipping. \\
$70048-13-20-00$ & 43099 & $52978.374306(2)^{2}$ & Double-peaked ingress. \\
$80040-01-06-00$ & 43342 & $53017.093350(3)$ & Normal eclipse. \\
$90059-02-01-00$ & 43684 & $53071.5869083(9)$ & Eclipse features sharp; High count rate. \\
$90059-02-02-00$ & 43685 & $53071.746244(3)$ & Eclipse features sharp; High count rate. \\
$90059-02-03-00$ & 43686 & $53071.9055842(6)$ & Eclipse features sharp; High count rate. \\
$90059-02-04-00$ & 43687 & $53072.064922(2)$ & Eclipse features sharp; High count rate. \\
\hline
\end{tabular}

\footnotetext{
${ }^{1}$ The fitted mid-eclipse times given to the first uncertain digit; The errors are in parenthesis for that digit.

${ }^{2}$ No adjustment is made in the fitted mid-eclipse time for the double-peaked ingress profile.
} 